\title{
Compiling gate networks on an Ising quantum computer
}

\author{
M. D. Bowdrey ${ }^{*}$ and J. A. Jonest \\ Centre for Quantum Computation, Clarendon Laboratory, \\ University of Oxford, Parks Road, OX1 3PU, United Kingdom \\ E. Knil声 \\ Mathematical and Computational Sciences Division, \\ National Institute of Standards and Technology, Boulder CO 80305

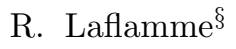 \\ Institute for Quantum Computing, University of Waterloo, ON, \\ N2L 3G1, Canada, and Perimeter Institute for Theoretical Physics, \\ 31 Caroline Street North, Waterloo, ON, N2L 2Y5, Canada
}

(Dated: October 30, 2018)

\begin{abstract}
Here we describe a simple mechanical procedure for compiling a quantum gate network into the natural gates (pulses and delays) for an Ising quantum computer. The aim is not necessarily to generate the most efficient pulse sequence, but rather to develop an efficient compilation algorithm that can be easily implemented in large spin systems. The key observation is that it is not always necessary to refocus all the undesired couplings in a spin system. Instead the coupling evolution can simply be tracked and then corrected at some later time. Although described within the language of NMR the algorithm is applicable to any design of quantum computer based on Ising couplings.
\end{abstract}

PACS numbers: 03.67.Lx

Quantum computers [1] have generated considerable interest in recent years due to their apparent ability to perform computations that are intractable on any classical computer. Although the construction of a general purpose quantum computer capable of solving real problems remains a challenge, preliminary results have been demonstrated in several systems 2], most notably nuclear magnetic resonance (NMR) [3, 4, 5, 6] and trapped ions $\mathbf{7}, 8,0$, 10]. When implementing simple quantum algorithms on devices with a small number of qubits, it is perfectly possible to design an implementation "by hand". With larger systems, however, this approach becomes impractical, and it is desirable to automate the process. Here we describe a simple procedure that allows a sequence of abstract quantum logic gates (the most common device-independent description of a quantum algorithm) to be compiled into a sequence of pulses and delays, which are the natural gates on an Ising quantum computer.

By an Ising quantum computer we mean a system with a background Hamiltonian

$$
\mathcal{H} / \hbar=2 \pi \sum_{i} \nu_{i} I_{z}^{i}+\pi \sum_{i<j} J_{i j} 2 I_{z}^{i} I_{z}^{j}
$$

where, following NMR notation [1], $I_{z}=\sigma_{z} / 2$ is the angular momentum operator in the $z$ direction, $\nu_{i}$ is the

\footnotetext{
*Electronic address: mark.bowdrey@physics.org

${ }^{\dagger}$ Electronic address: jonathan.jones@qubit.org

${ }^{\ddagger}$ Electronic address: knill@boulder.nist.gov

$\S$ Electronic address: laflamme@iqc.ca
}

precession frequency of the $i$ th qubit, and $J_{i j}$ is the Ising coupling strength between pairs of qubits; here we assume that all the $n(n-1) / 2$ Ising couplings in an $n$ qubit system are of significant size. This Hamiltonian arises naturally in NMR systems, where the qubits correspond to spin-half particles and the scalar $J$ coupling takes the Ising form within the weak coupling limit, but also occurs more generally. It can be simplified by working in a multiply rotating frame, where the frame used to describe each qubit rotates at the Larmor precession frequency $\nu_{i}$, so that the effective Hamiltonian contains only the coupling terms. We also assume that the system can be controlled by the application of arbitrary single-qubit gates to any qubit or group of qubits. In an NMR system these single-qubit gates would be implemented using resonant radiofrequency pulses, with individual qubits being distinguished by their unique Larmor frequencies. We refer to these single-qubit gates as pulses whether or not they are implemented in this way.

It is well known that any quantum algorithm can be implemented using only single-qubit and two-qubit gates, such as CNOT (controlled-NOT) gates [12, 13]. Furthermore, the CNOT gate can itself be decomposed in terms of (single-qubit) Hadamard gates and a controlled- $\sigma_{z}$ gate, which converts $|11\rangle$ to $-|11\rangle$ while leaving other states unchanged. Finally, a controlled- $\sigma_{z}$ is essentially equivalent to a $90^{\circ}$ evolution under an Ising coupling, differing only by single-qubit $z$ rotations [5]. These are most conveniently handled not by rotating the qubit, but rather by rotating its reference frame, a method usually known as abstract reference frames [14]. Similar techniques can be used to implement more general controlled phase-shift gates, which are useful for building such gates as the con- 


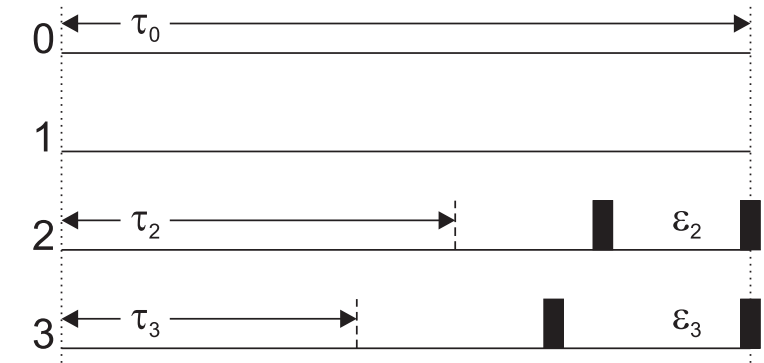

FIG. 1: An example of rescaling couplings between four qubits. The solid blocks indicate NOT gates $\left(180^{\circ}\right.$ pulses $)$.

trolled square-root-of-NOT [5]. Thus arbitrary quantum circuits can be constructed out of pulses and controlled evolutions under the Ising Hamiltonian. Here we simply assume that a circuit comprising only single-qubit gates and controlled phase-shift gates is available.

In order to achieve the desired evolutions it is necessary to sculpt the Hamiltonian into a more suitable form, in which only the desired couplings of the required strength are present. This is easily achieved by using spin echoes. Spin echoes are widely used in conventional NMR experiments 11] to refocus undesirable single-spin interactions, but they can also be used to effectively rescale Ising coupling terms within a Hamiltonian.

The basic procedure, which is based on the concept of an average Hamiltonian, is easily understood and is exemplified by Fig. 1 The coupling between qubits 0 and 1 evolves for the entire period, $\tau_{0}$, and so the average coupling strength experienced by these qubits is equal to the underlying coupling strength, $J_{01}$. The coupling from qubit 0 to qubit 2 is partially refocused by applying a pair of NOT gates $\left(180^{\circ}\right.$ pulses) to one of the two qubits involved. The effect of these NOT gates is to reverse the coupling evolution [15, 16] for a period of length $\epsilon_{2}$, and so it appears that the coupling has evolved only for a time $\tau_{2}=\tau_{0}-2 \epsilon_{2}$. Alternatively (and equivalently) the situation can be described as if the coupling had evolved for the whole time $\tau_{0}$ but with a reduced coupling strength $J_{02}^{\prime}=\left(\tau_{2} / \tau_{0}\right) J_{02}$. In the same way the coupling between qubits 0 and 3 has been rescaled by a factor of $\tau_{3} / \tau_{0}$, with $\tau_{3}=\tau_{0}-2 \epsilon_{3}$. The effective coupling strengths between other pairs of qubits can be worked out in a similar way. Clearly $J_{12}^{\prime}=\left(\tau_{2} / \tau_{0}\right) J_{12}$ and $J_{13}^{\prime}=\left(\tau_{3} / \tau_{0}\right) J_{13}$, by analogy with the couplings to qubit 0 . The last problem is to calculate the net evolution for the coupling between qubits 2 and 3, where NOT gates are applied to both qubits, and a little thought shows that the scaling factor is $\tau_{23} / \tau_{0}$ with a net evolution time $\tau_{23}=\tau_{0}-2\left(\epsilon_{3}-\epsilon_{2}\right)$.

The standard approach to date has been to use refocusing networks to sculpt the underlying Ising Hamiltonian into some ideal form, usually retaining only a small number of coupling interactions. In particular, efficient refocusing schemes are known [15, 16] to retain any one coupling in an extended network. There are, however, some limitations on the average Hamiltonians that can be achieved. For example, consider a system of three coupled spins, with a Hamiltonian containing three coupling terms: it is possible to keep any one of these terms while refocusing the other two, but it is not possible to keep two terms while refocusing the third. If such an average Hamiltonian is desired it is usually necessary to implement the two Hamiltonians sequentially. Designing evolution networks in large systems can become a complex business.

Our compilation algorithm provides an alternative approach for designing pulse sequences on Ising quantum computers with large numbers of spins. The aim is not to generate the most efficient pulse sequence, but rather to develop a simple algorithm that can be easily implemented in large spin systems. The key observation is that it is not, in fact, necessary to implement the exact Ising coupling evolutions shown in the network. In particular, it is not always necessary to refocus all the undesired Ising couplings in a spin system. Instead the coupling evolution can simply be tracked, and their values corrected at some appropriate time. This is because singlequbit gates applied to one qubit commute with couplings that do not involve this qubit. Thus it is only necessary to achieve the "correct" evolution for those qubits to which single-qubit gates are applied. Furthermore, since single-qubit gates applied to different qubits all commute with one another, two or more "simultaneous" singlequbit gates can in fact be applied sequentially in any order.

The first stage is to redraw the network so that singlequbit gates are applied sequentially, rather than in parallel. Simultaneous gates can be applied in any order; the simplest approach is to apply them from top to bottom. We refer to the qubit to which a single-qubit gate is applied as the target qubit, and any other qubits as control qubits. It is not necessary that evolution periods generate any particular coupling evolution between the control qubits, but these additional evolutions must be tracked through the circuit.

Couplings are tracked by recording the net evolution angle (modulo $360^{\circ}$ ) generated for each of the $n(n-1) / 2$ couplings at all significant points in the network, where the change in the evolution angle is given by

$$
\delta \theta_{i j}=\pi J_{i j}^{\prime} \tau,
$$

and $J_{i j}^{\prime}$ is the average coupling strength between qubits $i$ and $j$ during a time period $\tau$. It is essential that all the coupling angles involving a target qubit have the correct value as defined by the gate network before applying a single-qubit gate to the target. This is easily achieved by applying NOT gates to the control spins, thereby changing the average coupling strengths, so that all the couplings reach the desired angle in the same evolution time. When the single-qubit gate is applied to the target qubit, all coupling angles to this qubit are reset to zero. Couplings between control qubits are simply tracked throughout the process. 


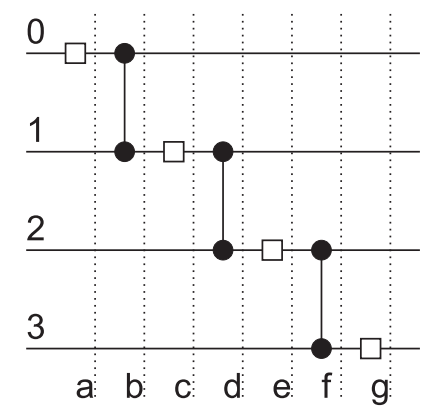

FIG. 2: An example quantum circuit, based on the algorithmic benchmark of Knill et al. [14]. White squares indicate $90_{y}^{\circ}$ pulses (pseudo-Hadamard gates) while black circles connected by control lines are Ising coupling gates with a target angle of $90^{\circ}$ between the coupled qubits.

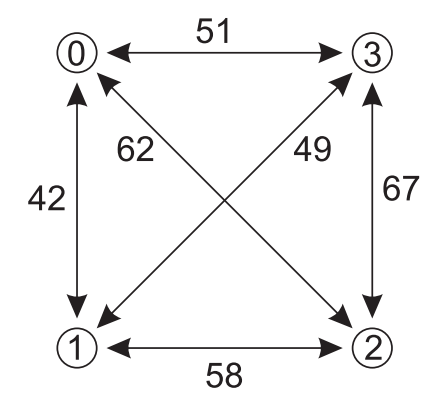

FIG. 3: An example Ising quantum computer, described by indicating the strengths of the Ising couplings (measured in $\mathrm{Hz}$ ) between all pairs of qubits; these couplings were chosen arbitrarily.

This procedure can be clarified by considering a concrete example, and here we consider the implementation of the quantum circuit shown in Fig. 2] on the Ising quantum computer depicted in Fig. 3 The circuit is based on the algorithmic benchmark of Knill et al. 14], but the coupling strengths shown in the Ising computer were chosen at random. The key values in an Ising computer are the coupling angles achieved between pairs of qubits, and these will be considered at the points marked with lower case letters in Fig. [2]

The first part of the circuit is simple: the system begins with all coupling angles set to zero, and the first single-qubit gate has no effect, so that at point (a) all coupling angles are $0^{\circ}$. By point (b) it is necessary that the coupling angle between qubits 0 and 1 be increased to $90^{\circ}$, while all other coupling angles to qubit 1 (which is the target of the next single-qubit gate) remain at zero; all further couplings can simply be tracked. This can be achieved by evolving under the coupling Hamiltonian for a time $1 / 2 J_{01}=11905 \mu \mathrm{s}$ with NOT gates applied to qubits 2 and 3 at the middle and end of the coupling periods, acting to completely suppress the couplings from these qubits to qubit 1 . The couplings from the other control qubits (2 and 3 ) to qubit 0 are also suppressed, but the coupling between qubits 2 and 3 will evolve for
TABLE I: Coupling evolution angles (measured in degrees, and rounded to the nearest degree) between every pair of qubits at various points in the initial part of the circuit shown in Fig. 2 Coupling angles indicated in boldface are coupling angles to target qubits that must be set correctly; other angles are simply being tracked as part of the algorithm.

\begin{tabular}{l|rrrrrrr} 
angle & $\mathrm{a}$ & $\mathrm{b}$ & $\mathrm{c}$ & $\mathrm{d}$ & $\mathrm{e}$ & $\mathrm{f}$ & $\mathrm{g}$ \\
\hline 0,1 & 0 & $\mathbf{9 0}$ & 0 & 70 & 70 & 70 & 70 \\
0,2 & 0 & 0 & 0 & $\mathbf{0}$ & 0 & 276 & 276 \\
0,3 & 0 & 0 & 0 & 0 & 0 & $\mathbf{0}$ & 0 \\
1,2 & 0 & $\mathbf{0}$ & 0 & $\mathbf{9 0}$ & 0 & 78 & 78 \\
1,3 & 0 & $\mathbf{0}$ & 0 & 76 & 76 & $\mathbf{0}$ & 0 \\
2,3 & 0 & 144 & 144 & $\mathbf{0}$ & 0 & $\mathbf{9 0}$ & 0
\end{tabular}

the whole coupling time, and thus achieve a coupling angle of $144^{\circ}$. The next step, at point (c), is to apply a single-qubit gate to qubit 1 ; at this point all coupling angles involving this qubit are reset to zero, while leaving all other angles unchanged. Thus the three coupling angles involving only control qubits still have the values $\theta_{02}=0^{\circ}, \theta_{03}=0^{\circ}$, and $\theta_{23}=144^{\circ}$. These (and further) evolutions are summarized in Table \.

The first interesting point in the algorithm occurs at (d). At this stage the algorithm requires the coupling angles to qubit 2 to be set correctly, that is $\theta_{02}=0^{\circ}$, $\theta_{12}=90^{\circ}$, and $\theta_{23}=0^{\circ}$. Allowing for the current values of the coupling angles, and recalling that coupling angles are defined modulo $360^{\circ}$, the additional evolution required is $\delta_{02}=0^{\circ}, \delta_{12}=90^{\circ}$, and $\delta_{23}=216^{\circ}$. Considering these evolutions individually, the coupling times required are $\tau_{02}=0 \mu \mathrm{s}, \tau_{12}=8621 \mu \mathrm{s}$ and $\tau_{23}=17910 \mu \mathrm{s}$. Clearly the last coupling requires the longest time, and so all the desired coupling angles can be achieved by evolving under the full coupling Hamiltonian for $8955 \mu \mathrm{s}$, with two couplings, $J_{02}$ and $J_{12}$, being (respectively) totally and partially refocused. Thus NOT gates should be applied to qubit 0 at the middle and end of the coupling period, while the first NOT gate is applied to qubit 1 after a time $\tau_{12}+\left(\tau_{23}-\tau_{12}\right) / 2=13266 \mu$ s, with the second NOT gate applied at the end. The remaining coupling angles can then be calculated by noting that $J_{03}$ is completely refocused, $J_{13}$ evolves for the same time as $J_{12}$, giving an angle of $76^{\circ}$, and $J_{01}$ evolves for a net time of $9288 \mu \mathrm{s}$, giving an additional coupling angle of $70^{\circ}$.

At point (e), a single-qubit gate is applied to qubit 2 , and so all coupling angles involving this qubit are reset to zero. The couplings at point (f) are then set using the same method as was used for (d). This time the limit coupling is $J_{13}$ which requires a time of $\tau_{13}=32200 \mu$ s to acquire an additional coupling angle of $\delta_{13}=284^{\circ}$. The coupling $J_{03}$ is completely refocused by applying NOT gates to qubit 0 at the middle and end of this coupling period, while the coupling $J_{23}$ must be partially refocussed by applying the first NOT gate to qubit 2 after a time of $19832 \mu \mathrm{s}$. The remaining couplings are tracked 


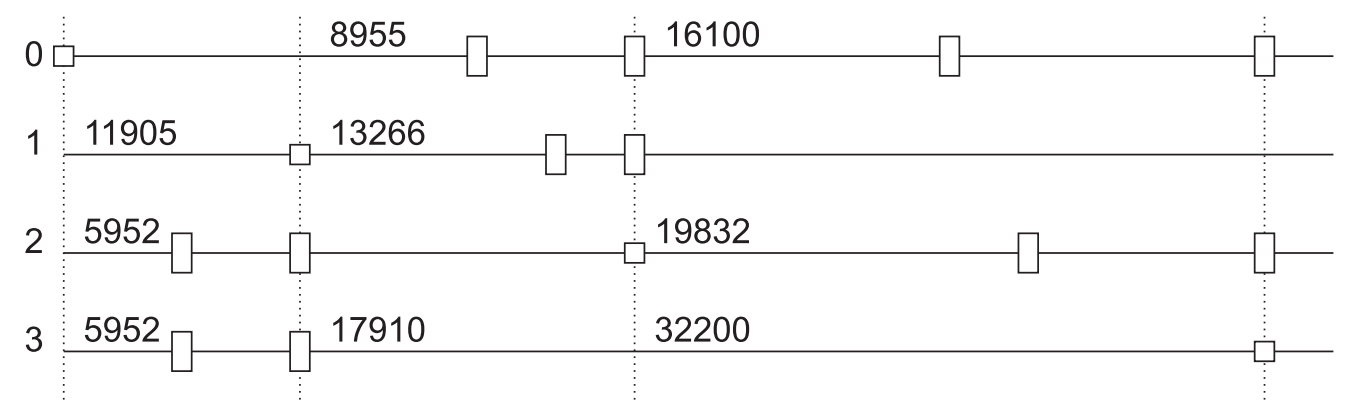

FIG. 4: Sequence of refocusing pulses used to implement the circuit shown in Fig. 2 on the Ising quantum computer shown in Fig. 3. White squares indicate $90_{y}^{\circ}$ pulses as before, while white rectangles indicate NOT gates. These single-qubit gates are assumed to be instantaneous. The evolution periods are drawn approximately to scale, and the lengths of some periods (measured in $\mu \mathrm{s}$ ) are indicated.

as usual, and their values are shown in Table \ The complete sequence of refocusing pulses and single-qubit gates is shown in Fig. [4 Note that the evolution times depicted are those calculated above, which include the effects of rounding errors; exact calculations would give slightly different times.

As discussed above, this algorithm is not necessarily intended to produce the most efficient pulse sequence, but it is still necessary to check that the simplicity of implementation is not achieved at an excessive cost either in pulse sequence length or in the number of pulses required. Clearly the complexity of a pulse sequence will depend on details of the circuit being implemented and the range of coupling strengths in the experimental system. It is, however, simple to consider some limiting situations.

Any portion of a circuit in a system of $n$ qubits can be classified according to the number $p$ of target qubits involved, which obviously lies between the extreme values of 1 and $n$. Using our new algorithm this section will be divided into at most $p$ separate sections, each of which will require $2(n-2)$ refocusing pulses; in total the implementation will require less than $2 \times n \times p$ pulses. Using the Hadamard matrix approach will require between 1 and $p$ refocusing periods (depending on exactly which couplings have to be controlled), each of which will contain approximately $n^{2}$ refocusing pulses [16]. The relative efficiency of our new algorithm against the Hadamard algorithm will depend on the number of refocusing periods required by the latter. In a system such as that shown in Fig. [3] where all the coupling strengths are different, it will not be possible in general to combine different evolution periods, and so the Hadamard approach will require about $p \times n^{2}$ pulses. Thus our new algorithm will normally use fewer pulses than previous methods. The time required to implement a set of gates is another important consideration, but once again will vary greatly from network to network. It is easy to see that pulse sequences produced by our new algorithm will in the worst case take $p$ times longer than those produced by current methods, although it seems likely that the relative performance will be better than this in real situations.

It is possible to imagine a large number of ways in which this algorithm could be extended, resulting in simpler or shorter pulse sequences. Here we confine ourselves to two particularly simple extensions, both of which can be easily implemented. Firstly, we note that it is not in fact necessary to place our pairs of NOT gates at the end of the coupling period, as shown in Fig. 1 Instead the pair of refocusing gates can be placed at any point within the coupling period without affecting the net coupling to the target spin (the position will, of course, affect the net couplings to other control spins). In particular, we can choose to place the first NOT gate at the beginning of a coupling period, and if this period is preceded by one with a NOT gate at the end then two of the four NOT gates can be canceled.

Secondly, as described so far, it has been necessary to set coupling evolution angles to a target spin to the correct value modulo $360^{\circ}$; thus qubit pairs can, in principle, be required to undergo evolutions of up to $360^{\circ}$ to achieve the desired angle. This is, however, excessive. We note that

$$
\exp \left[-i \pi 2 I_{z}^{j} I_{z}^{k}\right]=\exp \left[-i \pi\left(I_{z}^{j}+I_{z}^{k}\right)\right]
$$

and so a $180^{\circ}$ coupling evolution on a pair of qubits is equivalent to a $180^{\circ}$ frame rotation applied to both qubits. Thus it is never necessary for qubits to undergo an evolution through more than $180^{\circ}$, as any additional evolution can be achieved through frame rotations.

Thirdly, we note that, in addition to partly refocusing coupling periods, NOT gates can also be used to negate the sign of an Ising coupling. If a pair of NOT gates is applied to a control qubit at the beginning and end of an evolution period, then the evolution of the Ising coupling will be reversed throughout this period. If NOT gates are already applied to the qubit during this period to partially refocus a coupling, then two of the four NOT gates can be cancelled, so there will be no overall increase in the number of gates. Combined with the previous observation, we note that any desired evolution can be achieved using a coupling evolution in the range $\pm 90^{\circ}$. This allows the implementation time to be reduced by a factor of around four, at the cost of a small increase in the number of NOT gates. 
Finally, we note that it is possible to combine the traditional approach and our new algorithm to produce hybrid schemes. For example, it might be useful to compile portions of a network independently of any larger computation, in effect treating them as subroutines. To do this, it is necessary to ensure that all the couplings in the system have correct evolution angles at the end of the subroutine, not just those involving the final target qubit. This can be achieved by ending the network with a conventional refocusing period. If, however, the whole network is compiled together, then this final refocusing period will normally not be required. Computations usually end with measurements on some of the qubits in the computational basis, and these eigenstates commute with the Ising Hamiltonian. The results obtained from measurements of these qubits are, therefore, independent of their couplings angles, and it is not necessary to refocus couplings to them.

\section{Acknowledgments}

MB and JAJ thank the UK EPSRC for financial support. RL would like to thanks MITACS and ARDA. Contributions to this work by NIST, an agency of the US government, are not subject to copyright laws.
[1] C. H. Bennett and D. P. DiVincenzo, Nature 404, 247 (2000).

[2] S. L. Braunstein and H.-K. Lo, Scalable Quantum Computers: Paving the Way to Realization (Wiley, 2001).

[3] D. G. Cory, A. F. Fahmy and T. F. Havel, in "PhysComp '96' (T. Toffoli, M. Biafore and J. Leão, Eds.), pp. 87-91, New England Complex Systems Institute (1996)

[4] D. G. Cory, A. F. Fahmy, and T. F. Havel, Proc. Natl. Acad. Sci. USA 94, 1634 (1997).

[5] J. A. Jones, Prog. NMR Spectrosc. 38, 325 (2001).

[6] L. M. K. Vandersypen, M. Steffen, G. Breyta, C. S. Yannoni, M. H. Sherwood, and I. L. Chuang, Nature 414, 883 (2001).

[7] C. Monroe, D. M. Meekhof, B. E. King, W. M. Itano and D. J. Wineland, Phys. Rev. Lett. 75, 4714 (1995).

[8] S. Gulde, M. Riebe, G. P. T. Lancaster, C. Becher, J. Eschner, H. Häffner, F. Schmidt-Kaler, I. L. Chuang, and R. Blatt, Nature 421, 48 (2003).

[9] M. Riebe, H. Häffner, C. F. Roos, W. Hänsel, J. Benhelm, G. P. T. Lancaster, T. W. Körber, C. Becher, F. Schmidt-
Kaler, D. F. V. James and R. Blatt, Nature 429, 734 (2004).

[10] M. D. Barrett, J. Chiaverini, T. Schaetz, J. Britton, W. M. Itano, J. D. Jost, E. Knill, C. Langer, D. Liebfried, R. Ozeri and D. J. Wineland, Nature 429, 737 (2004).

[11] R. R. Ernst, G. Bodenhausen, and A. Wokuan, Principles of Nuclear Magnetic Resonance in One and Two Dimensions (Clarendon Press, Oxford, 1987).

[12] A. Barenco, C. H. Bennett, R. Cleve, D. P. DiVincenzo, N. Margolus, P. Shor, T. Sleator, J. Smolin and H. Weinfurter, Phys. Rev. A 52, 3457 (1995).

[13] D. P. DiVincenzo, Proc. R. Soc. Lond. A 454, 257 (1998).

[14] E. Knill, R. Laflamme, R. Martinez, and C.-H. Tseng Nature 404, 368 (2000).

[15] J. A. Jones and E. Knill, J. Magn. Reson. 141, 322 (1999).

[16] D. W. Leung, I. L. Chuang, F. Yamaguchi and Y. Yamamoto, Phys. Rev. A 61, 042310 (2000). 